\title{
Development and Implementation of Constant Friction Power Control in a Reduced Scale Brake Dynamometer for Investigations of Automotive Brake Systems
}

\author{
Stephan Raczek and Georg-Peter Ostermeyer \\ Institute of Dynamics and Vibrations, TU Braunschweig, Braunschweig 38106, Germany
}

\begin{abstract}
For tribological investigations of automotive brakes, both full and reduced scale brake dynamometers are used. Full scale brake dynamometers test the entire brake system, including caliper, brake pad, brake disk, wheel suspension, etc. The AUT (automated universal tribotester) is a reduced scale brake dynamometer with a pin on disk test configuration. A brake pad specimen is used as the pin and a brake disk is used as the counter body. Compared to full scale brake dynamometers, the AUT encounters fewer influences from the test systems themselves. The AUT was developed at the Institute of Dynamics and Vibrations in Braunschweig. It can be used for high frequency analyses of the coefficient of friction, and investigations of the boundary layer between brake pad and disk, along with the associated NVH behaviors. A digital camera and two laser triangulation sensors are used for optical investigations of the brake pad and brake disk $[1,2]$. The focus of this paper is the development of a new control feature of the AUT. The AUT employs a compactRIO from National Instruments as its measurement and control device. The velocity and position control loops are implemented in the compactRIO's FPGA along with high frequency synchronous measurement data acquisition. Its real-time controller coordinates the measurement and the storage of measured data. The test device has three degrees of freedom: normal load, sliding speed, and temperature, all of which can be defined by the user for each brake application. New control strategies based on the friction force as a feedback parameter allow for the implementation of new features. In this paper, the realization of measurements with constant friction power throughout the friction procedure will be shown. This new degree of freedom in the friction measurements enables investigations to be carried out in which it is necessary to bring a specific energy in the friction process. In this paper, a p-controller on the real-time system calculates the required set point for the position control loop. The new feature is verified through a benchmark test [6]. Based on the control loop with the friction force as the feedback parameter, it is also possible to emulate in-stop brakes with virtual inertia. In this way, many other types of full scale brake dynamometers can be simulated. The long-term aim is the identification of the influence of test devices on the test results.
\end{abstract}

Key words: Automated universal tribotester, pin-on-disk, friction power, tribological investigations.

\section{Nomenclature}

$\begin{array}{ll}\text { AUT } & \text { Automated universal tribotester } \\ \text { COF } & \text { Coefficient of friction } \\ \text { FPGA } & \text { Field programmable gate array } \\ \text { NVH } & \text { Noise, vibration, harshness }\end{array}$

\section{Introduction}

For tribological investigations of vehicle brake systems, specialized test devices are often used. These devices are known as full scale brake dynamometers.

Corresponding author: Georg-Peter Ostermeyer, Prof. Dr.-Ing. habil., research fields: drilling and reservoir dynamics, brake dynamics, NVH, "clean" braking, self-organization, and self-synchronization in complex dynamic systems.
They consist of a large rotating body, which represents the total vehicle mass during the braking process, and the entire brake system, including pads, rotor, caliper, wheel suspension, and so on. Such devices are suitable for performance tests, but in order to gain insights into the tribological phenomena in the boundary layer between the brake pad and rotor, a highly specialized device is necessary. Reduced scale brake dynamometers are Pin-On-Disk test devices that aid in this pursuit. Such devices are optimized based on the requirements of the vehicle brake system and investigate a small specimen rather than an entire brake pad. 
The Institute of Dynamics and Vibrations in Braunschweig (Germany) has developed two reduced scale brake dynamometers. One of these is the AUT (automated universal tribotester). The main components of this test device are two linear stages, a rotary drive, and a load unit. Several types of specimens can be used; the friction surface of a typical brake pad material test sample is between 200 and 400 $\mathrm{mm}^{2}$ in size. The maximum speed of the rotary drive is $1500 \mathrm{RPM}$. The continuous torque limit is $65 \mathrm{Nm}$ over the entire speed range.

Fig. 1 shows a schematic image of the elementary components of the AUT. Actuator components are shown in green and the measurement components are shown in red.

A fundamental tribological measurement parameter is the COF (coefficient of friction). On the AUT, a 3D force sensor mounted behind the brake pad specimen measures the forces near to the tribological contact. These signals are used to calculate the COF. To understand the interaction between the brake disk and the pad specimen, it is not sufficient to only measure the COF under various boundary conditions. There are also, for example, dynamic wear processes and wear particle dynamics on both surfaces. The automation of the AUT allows for quasi in situ optical investigations of the disk and pad topographies. This is carried out between measurements, while the test samples remain mounted on the device. Results from these

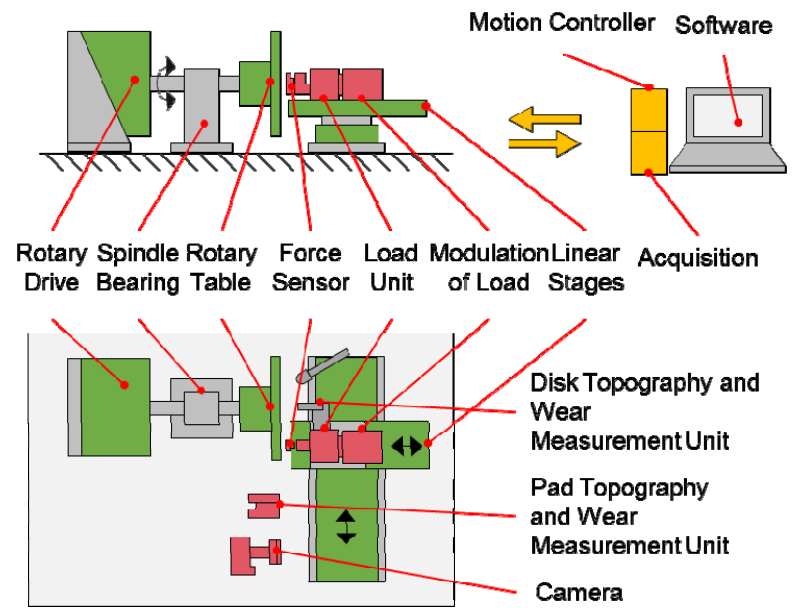

Fig. 1 Schematic sketch of the AUT (edited from [2]). investigations which include measurements of the wear, the surface roughness and particle reservoirs in the surface. An acoustic unit is used to detect NVH-phenomena. Fig. 2 shows the AUT without the heating chamber, which when mounted encases the brake disk [2].

\section{Measurement and Control Device}

The most crucial component for the automation of the AUT is the measurement and control device. It fulfills the requirements of synchronous high frequency measurement data acquisition and the control of the AUT's drives. The AUT's actuators include one rotary drive, two spindle-driven linear stages, one linear stage driven by a magnetic actuator, and a voice coil actuator. The closed loop control of the rotary drive and both spindle-driven linear stages is implemented in the measurement and control device [6].

Because the AUT must be flexible for new features and specialized measurement tasks in the future, its measurement and control device requires a modular design. The compactRIO (NI cRIO-9074) from National Instruments fulfills all requirements for the measurement and control device. Consideration of this device can be separated into three parts:

\subsection{IO Modules}

The compactRIO has eight slots for several IO modules. National Instruments offers various modules, for example analog inputs, temperature inputs, digital IOs, and modules with an RS422 interface. The number of modules for the AUT depends on the requirements shown in Table $1[3,4]$.

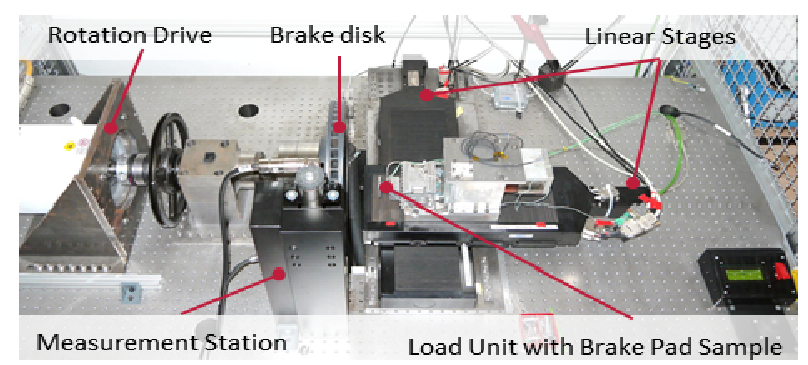

Fig. 2 Photograph of the AUT (without heating chamber) (edited from [6]). 
Table 1 Requirements of the IO modules.

\begin{tabular}{llll}
\hline Signal & Direction & \multicolumn{2}{l}{ Sample frequency } \\
\hline \multirow{2}{*}{ Analog } & Input & $910 \mathrm{kHz}$ & $>10 \mathrm{kHz}$ \\
& Output & 4 & 4 \\
& Input & 8 & 0 \\
Digital & Output & 11 & 7 \\
& Bidirectional & 1 & 0 \\
\hline
\end{tabular}

\subsection{FPGA}

The FPGA is a reconfigurable logic circuit, which is optimized for high speed operations. It consists of logic blocks, block RAM, multipliers, and IO blocks. The clock speed varies between 10 and $100 \mathrm{MHz}$, depending on the complexity of the FPGA configuration. In this case, the FPGA has two major tasks: synchronous data acquisition and the closed loop control of the rotary drive and the two spindle-driven linear stages $[3,4]$.

\subsection{Real-Time Controller}

The third component of the compactRIO is a real-time controller, which is necessary for the reliability of the test device. It is responsible for open loop control and data storage.

With respect to the friction process, the AUT has three degrees of freedom. Before the measurement, it can heat the brake disk to a maximum temperature of $200{ }^{\circ} \mathrm{C}$ using a heating chamber. During the measurement, the normal force and the rotation speed can be either held constant, ramped, or modulated.

Several signals with various sampling rates are synchronously recorded. The sampling frequency varies between $50 \mathrm{kHz}$ and $2 \mathrm{~Hz}$ (Fig. 3). Optical measurements of the pad and disk surface can be performed before and/or after each brake application.

Fig. 4 shows an overview of the AUT software. It begins with the initialization of the axis, followed by a loop over various parameter sets. The normal load of the AUT is not applied using load control, but rather by displacement control.

Therefore, the AUT intermittently measures the

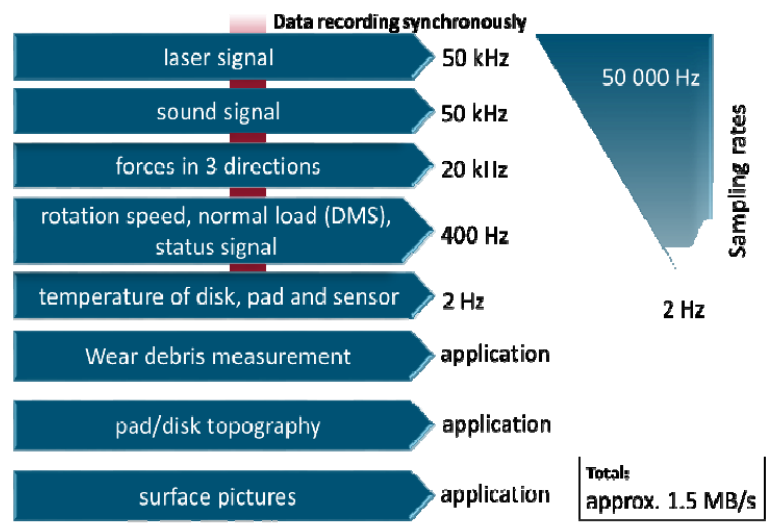

Fig. 3 Signals of the AUT.

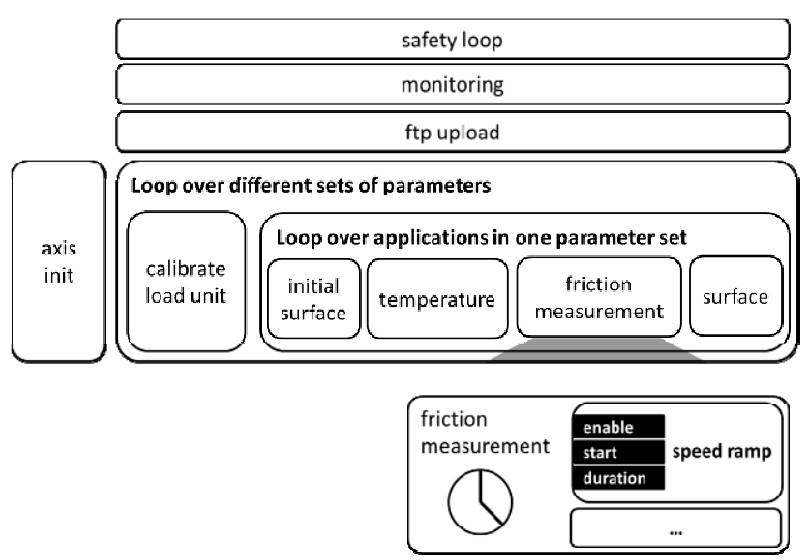

Fig. 4 Software design [6] (edited from [6]).

load-displacement curve of the load unit and calculates the corresponding stiffness. Afterwards, the AUT iterates over a loop of brake applications in one parameter set. After initial surface measurements, the AUT waits for the desired initial surface temperature to be reached before the friction measurement starts. The friction measurement is controlled by an internal clock. Each event, such as speed ramp or force application, has three general properties: an enable signal, start time, and duration. By using these signals, flexibly designed brake applications can be realized [6].

\section{Friction Power Control}

As already stated, the AUT can adjust the normal load and the sliding speed during brake applications. Typically, this is controlled by the user, for example, for a speed ramp with a constant normal load. For 
some investigations, it is necessary to drive the friction test with a specific friction power. To this end, other measurement systems often determine the required sliding speed and the normal load based on an assumed coefficient of friction. As the coefficient of friction is a dynamic parameter of the system, this could result in erroneous friction power levels and a friction power that varies throughout the measurement application.

The blue line in Fig. 5 represents the results achieved without using such a technique for constant friction power. The desired results, however, should be similar to the red line. New control loops are implemented in the AUT, which use the measured friction force, normal force and sliding speed as feedback parameters to solve this problem.

The friction power is calculated as the product of the sliding speed and friction force, and the friction force is calculated as the product of the coefficient of friction and the normal force (Eq. (1)).

$$
\boldsymbol{P}_{\text {fric }}=\boldsymbol{\mu} \cdot \boldsymbol{F}_{\text {Normal }} \cdot \boldsymbol{v}_{\text {Slide }}=\text { constant }
$$

As the coefficient of friction cannot be directly controlled, there are two potential ways to achieve a constant friction power: by adjusting the sliding speed or the normal force. Based on the results of the first benchmark test shown in the following section, the focus of the present work is the latter option. The AUT uses a proportional controller, which calculates the new set point for the normal force with respect to the difference between actual and target friction powers (Eq. (2)).

$$
\begin{array}{r}
F_{\text {Normal,target, } t}=\left(P_{\text {fric,target }}-P_{\text {fric,actual }}\right) \\
\cdot \boldsymbol{k}+\boldsymbol{F}_{\text {Normal,target, }-1}
\end{array}
$$

Fig. 5 Schematic of an investigation of a parameter with respect to the friction power.
The RT controller buffers a specific number of elements in two FIFOs in order to calculate the current sliding speed and friction force. The estimated setpoint for the normal load is used in combination with the load-displacement curve as the new setpoint for the position controller of the linear stage in the direction normal to the contact surface.

\section{Experimental Results}

As benchmarks for the new control strategy, two tests have been performed on the AUT:

- Three specific levels of friction power with different control strategies;

- Complex test matrix with four friction power levels and four input velocities.

As already stated, there are two ways to adapt the friction power. The strategies of adjusting the normal load and adjusting the sliding speed have been tested, in order to determine which strategy performs better. Fig. 6 shows the friction power at three specified levels $(75,150,225 \mathrm{~W})$.

At each power level, 90 brake applications with durations of 15 seconds were performed. Here, the variation of the friction power with the adaption of the normal is load minimal, so this strategy is implemented in the AUT. These results depend on the time constants of the AUT's drives and the high sensitivity of changes of the sliding speed to the coefficient of friction, and thereby to the friction power.

Fig. 7 further exemplifies the precision of the normal load adjustment strategy through a single brake application with $200 \mathrm{~W}$ friction power.

One second after applying the normal load, the AUT's friction power control is activated. As the coefficient of friction increases, the AUT reduces the normal load. As seen in Fig. 7a, there is a small overshoot at the beginning. During the friction process, the friction power is held nearly constant (Fig. 7b). Currently, only a P-controller is implemented. The overshot at the beginning can be reduced by using a PI 


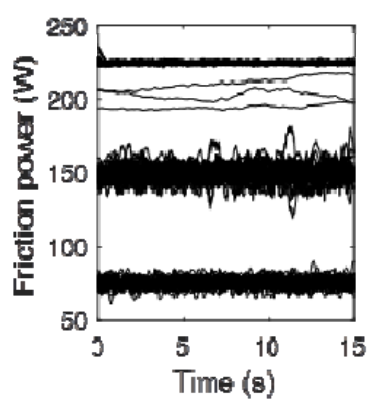

(a)

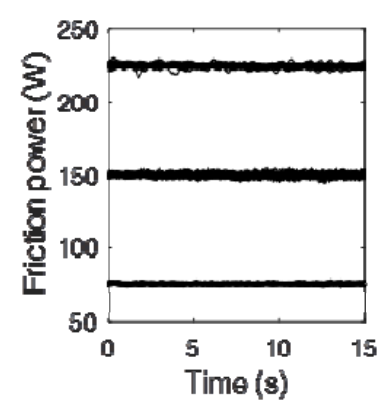

(b)
Fig. 6 Results of the benchmark test: (a) adjusting of sliding speed and (b) adjusting of normal load.

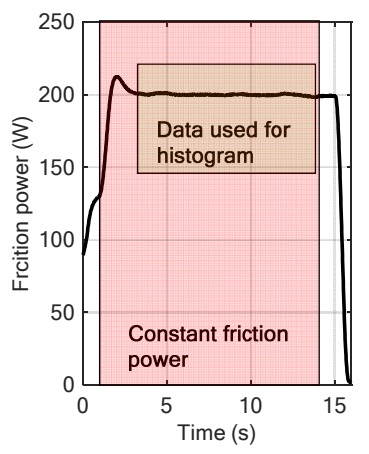

(a)

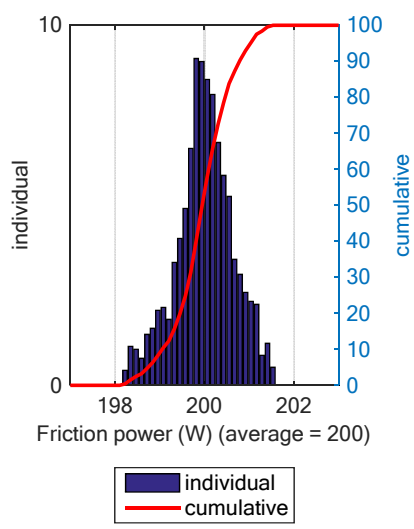

(b)
Fig. 7 Measurement results of one single measurment: (a) Friction power over time and (b) histogram of friction power.

or PID-controller. This is planned for further developments.

Systematic tribological investigations of a specific friction power are enabled by the new control loop shown above. The second benchmark test is a test matrix with four friction power levels and four sliding velocities. The normal load at the beginning of each brake application is calculated using Eq. (1) and an assumed coefficient of friction of 0.35 . In this case, the tribological parameters investigated are the coefficient of friction and the brake pad wear. A specialized measurement unit for pad topography is implemented in the AUT [5].

The wear is shown as a function of friction power in Fig. 8a. The wear measurements were carried out at the end of each 30 application parameter set block. It is clear that the wear increases with increasing friction

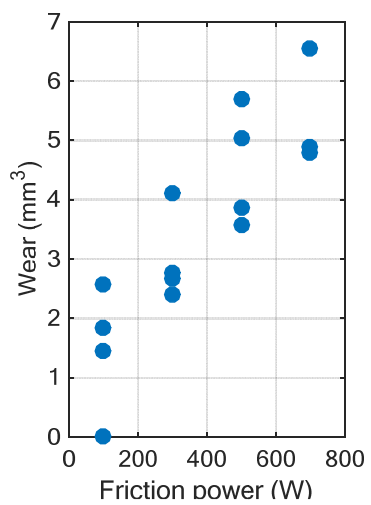

(a)

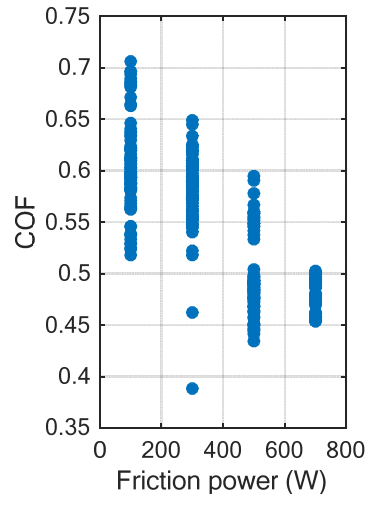

(b)
Fig. 8 (a) Wear and (b) coefficient of friction vs. friction power.

power. The average of the coefficient of friction for each brake application is shown in Fig. 8b. Here, the coefficient of friction decreases with increasing friction power. It is of interest to note that the coefficient of friction nearly reaches a value of 0.7 at low friction power. Considering the ideal constant friction power shown in Fig. 5, the friction power in these results remains relatively constant during the measurements, enabling such a systematic investigation. Typically, these types of investigations encompass a large number of input and output parameters. Here, the friction power and sliding speed are the input parameters; the resulting coefficient of friction, temperature and wear are output parameters.

Fig. 9 combines these data in one single plot. The $\mathrm{x}$ - and $\mathrm{y}$-axes represent the sliding speed and the friction force, respectively. The x-components of the arrows' lengths represent a normalized coefficient of friction; the y-components represent a normalized value of wear. Each arrow in the plot is the average of the results of 30 measurements with identical input parameters. The temperature during the brake application is denoted by the arrow's color. The aforementioned selection of the $\mathrm{x}$ - and y-axes allows all brake applications with the same friction power level to be plotted along respective constant friction power curves in the diagram. As shown in Fig. 8b, the maximum coefficient of friction occurs at $100 \mathrm{~W}$ friction power. In this new diagram, it is clear that a 


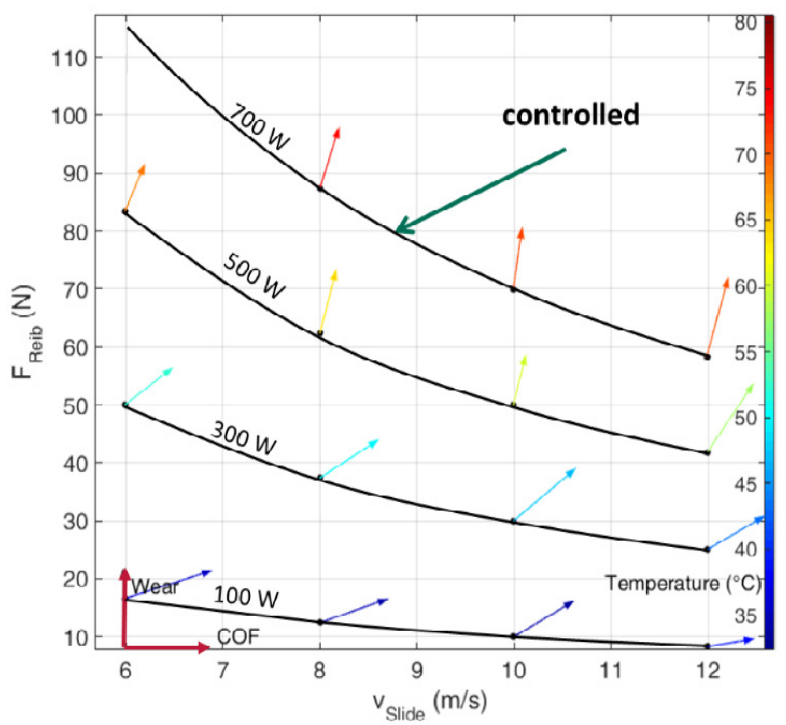

Fig. 9 Example measurement results.

low friction power and low speed result in a high coefficient of friction.

\section{Conclusion and Outlook}

This paper presented the AUT as a tribological test device with a pin-on-disk setup. It is used for investigations of automotive brakes. Along with common test features (constant sliding speed and normal load), advanced features such as speed ramps, load modulations, and optical investigations can be implemented. Here, the implementation of a new feature was presented: constant friction power. This feature uses the friction force and the sliding speed as feedback parameters. To hold the friction power constant, the normal load or the sliding speed must be adjusted. Based on the results of a benchmark test, the adjusting of the normal load was implemented. The p-controller was tested a with realistic test matrix. By using this new feature, it is possible to bring a specified friction power into the testing process. This correlates with the wear of the brake disk and pad. This technique introduces a wide range of potential investigations towards gaining a better understanding of the tribological boundary layer.

As further work, the controller can be improved by using a PI or PID-controller to reduce the overshoot observed in Fig. 7b. Regardless of the overshoots, however, the P-controller fulfilled the requirements of the current study.

Further work will also include implementation of virtual inertia properties, virtual car properties, and virtual scaling properties in the AUT. Based on control loops with the friction force as a feedback parameter, it is possible to emulate many other types of full scale brake dynamometers. The long term aim is the identification of the influences of the test devices on the test results.

\section{References}

[1] Ostermeyer, G.-P., and Perzborn, N. 2011. "Dynamic Friction Measurements, Especially for High Power Application." SAE technical paper 2011-01-2373. doi: 10.4271/2011-01-2373.

[2] Ostermeyer, G.-P., Schramm, T., Raczek, S., Bubser, F., and Perzborn, N. 2015. "The Automated Universal Tribotester.” Eurobrake 2015 Dresden, EB2015-STQ-015.

[3] National Instruments: CompactRIO Integrated Systems with Real-Time Controller and Reconfigurable Chassis-NI cRIO-907x, Data Sheet, Last Revised: 2014-11-06 07:14:18.0.

[4] National Instruments: CompactRIO Developers Guide, 05.2009.

[5] Schramm, T., and Ostermeyer, G.-P. 2016. "Automated Brake Pad Surface Topography Measurement Using the AUT.” Eurobrake 2016 Milan, EB2016-SVM-023.

[6] Raczek, T., and Ostermeyer, G.-P. 2016. "On the Software Development for a Reduced Scale Brake Dynamometer." Eurobrake 2016 Milan, EB2016-SVM-056. 